\title{
Detection of Ship Wakes in SAR Images Using Grayscale Parallel Coordinate Transform
}

\author{
Guozheng Yang \\ Dept. of Electronics Engineering, Tsinghua University, \\ Beijing 100084, China; Beijing Institute of Remote Sensing \\ Information, Beijing 100192, China \\ gzyangcn@gmail.com
}

\author{
Hongbing Ma, Weidong Sun \\ Dept. of Electronics Engineering, Tsinghua University \\ Beijing 100084, China \\ hbma@tsinghua.edu.cn \\ wdsun@tsinghua.edu.cn
}

\begin{abstract}
The detection of ship wakes is one of the most important targets in the area of SAR image applications, but the existing Radon Transform (RT) or Hough Transform (HT) based line detection methods can only be used to examine the collinearity of image points in the parametric space. Considering the characteristics of speckle noise and the spatial distribution of ship wakes along a line with certain width in SAR images, a new method using a nonparametric Grayscale Parallel Coordinate Transform (GPCT) is proposed. In this method, the original grayscale SAR image will be transformed into a parallel coordinate space by a new extended nonparametric GPCT, and the obvious points with high accumulation value will be detected by a modified iterative thresholding method. Then, the detected obvious points will be clustered according to its spatial distribution, and the center of each class will be determined to evaluate the lines of ship wakes. Experimental results using synthetic and real SAR images show that comparing with RT and HT based methods, our proposed method performs better in terms of the accuracy and correctness, better or equivalent in terms of the time efficiency.
\end{abstract}

Index Terms-SAR image, detection of ship wakes, Parallel Coordinate Transform

\section{I.INTRODUCTION}

Sailing in the sea, ships cause wakes behind them. The patterns of ship wakes imply size, heading direction and sailing speed information about ships [1], so we can get these implications and other useful information by detecting ship wakes. In SAR images, ship wakes tend to be V-shaped lines or strip lines. So, the detection of ship wakes in SAR images is equivalent to that of the line detection. In this respect, Radon Transform (RT) and Hough Transform (HT) are two of the most effective methods which can be considered [1]. A great deal of research is focused on RT or HT based methods for the detection of ship wakes in SAR images, such as [1] [4]. The main idea of these methods is to transform SAR images into the parametric space, and detect the lines by their collinearity.

But, [5] indicates that RT or HT based methods can only examine the collinearity of image points, but not care about the distribution of points along a line with certain width. This means that when detecting a line with certain width in noisy images, one may get wrong results. So, [5] proposes a new line detection method based on the Parallel Coordinate Transform (PCT) for noisy images. PCT is actually a nonparametric transformation method, and it will transform any point from image space into parallel coordinate space without supposing the point is on a line expressed by the parameterized form. This means when detecting lines with a width, it will be more effective and more efficient than the RT or HT based methods. [6] also uses the PCT to detect lines, but as same as [5], it binarizes the original grayscale images firstly. [7] indicates that the binaryzation of grayscale images may cause the loss of grayscale information, even change the spatial relationship and statistic characteristics between pixels.

Facing the above problems, we will extend the traditional PCT from binary into the grayscale transformation to deal with grayscale SAR images directly, and use clustering techniques to detect ship wakes in the noisy SAR images with certain width. This paper is organized as follows. Section II introduces the new concept of grayscale PCT and proposes our new 
method based on it, Section III compares our methods with other 2 methods using several synthetic and real SAR images, and the conclusions are drawn in Section IV.

\section{GPCT BASED DETECTION METHOD}

It is well known that the axes of the Cartesian Coordinate System (CCS) are vertical mutually. If placing them in the same direction but spacing a certain distance, we can get a new frame named the Parallel Coordinate System (PCS). At this time, (1) a point in the CCS will be mapped into a straight line in the PCS; (2) all points on a line in the CCS will be mapped into a group of straight lines in the PCS, which will intersect at one point if the slope of the line in the CCS is negative; (3) a point in the PCS will be mapped back into a straight line in the CCS as well [6]. The first two characteristics are the principle of PCT, whose implementation is an accumulative process.

As discussed above, traditional PCT can only deal with binary images but it is unable to process grayscale images, just as the methods proposed in [5] and [6]. This may cause the loss of grayscale information, and even for the HT based methods, a gray-scaled normalization is great helpful for the detection of ship wakes [2]. Here following the above idea, we extend the traditional PCT from binary into the grayscale transformation to deal with grayscale SAR images directly, whose steps are as following, (1) define $A(\xi, \eta)$ to note accumulated values at point $(\xi, \eta)$ of the PCT domain; (2) define $L(\xi, \eta)$ to note accumulated times at point $(\xi, \eta)$ in the PCT domain; (3) let the $A(\xi, \eta)$ be divided by the $L(\xi, \eta)$. When finishing these 3 steps, we get a gray-scaled PCT domain, which is named as Grayscale Parallel Coordinate Transform (GPCT).

We propose a new method based on the GPCT to detect ship wakes in SAR images. The flow consists of 3 steps, (1) transforming the original grayscale SAR images by the GPCT, and getting 2 transform domains; (2) preserving obvious points of the 2 GPCT domains with a modified iterative thresholding method; (3) clustering these points with the Hierarchical Agglomerative method [8] and determining centers of every clusters to locate the centerlines of the ship wakes. With these centers, we can furthermore retrieve size, heading direction and sailing speed information about the ship, and we also can evaluate the detective accuracy of our new method by transforming them back into the image domain.
As for step (2), the existing methods mostly set thresholds manually. We use a Neighborhood Valley Emphasis method presented in [9] to promote the automatic level of our method.

\section{EXPERIMENTS AND ANALYSES}

3 experiments using different synthetic and real SAR images are carried out on a PC with $1.8 \mathrm{GHz}$ CPU, 4GB RAM, Windows 7 and MATLAB r2012b to evaluate our new method, and the experimental results are compared with other 2 related methods.

Expt. 1 uses 3 synthetic data shown as background images in Fig. 1. The size of each image is $150 \times 150$ pixels, the width of every line in each image is 3 pixels, the mean of speckle noises in each image is 0 , but the variances of speckle noises in them are $0.03,0.18$, and 0.27 respectively. The purpose of Expt. 1 is to analyze the impacts of speckle noise level on the 3 methods. Expt. 2 uses 3 another synthetic data shown as background images in Fig. 2. The size of each image is still $150 \times 150$ pixels, the mean of speckle noises in each image is also 0 , the variance of speckle noises in each image is 0.18 , but the line widths in them are 3,5, and 7 pixels respectively. The purpose of Expt. 2 is to analyze the impacts of line width on the 3 methods. Expt. 3 uses 58 real SAR images, the size of each image is $150 \times 150$ pixels, line widths and speckle noise levels in each image are different. The purpose of Expt. 3 is to evaluate whether our method is better than other 2 methods under the real conditions.

The compared 2 related methods use the same flow as ours, and 4 indices are used here as the comparison metrics, which are number of False Alarms (FA), number of Miss Detections (MD), Runtime Efficiency (RE), and average of Normalized Distance (ND). The unit of the RE is second, and the ND is calculated by the following formula,

$$
N D=\sum_{i=1}^{N} \sqrt{\left(x_{i}^{*}-x_{i}\right)^{2}+\left(y_{i}^{*}-y_{i}\right)^{2}} /(N \times D I A G)
$$

where, $\left(x_{i}, y_{i}\right)$ and $\left(x_{i}^{*}, y_{i}^{*}\right)$ are the manually selected and algorithm selected centers in the transform space respectively, $\mathrm{N}$ is the number of clusters, and DIAG is the diagonal length of the transform space. Here, the manually selected centers by experts will be used as the ground truth. The smaller the ND is, the higher the accuracy of the detection method is. If there is no $\left(x_{i}^{*}, y_{i}^{*}\right)$ corresponding to $\left(x_{i}, y_{i}\right)$, ND will be set to1. 
In the first 2 experiments, Frequency of Thresholding (FT) is set to 3 , and the radius of clustering is set to 20 pixels. The line detection results of our method of Expt. 1 are shown in Fig. 1 with red color, and the averages of 4 indices of our method and other 2 methods are listed in Table I. The line detection results of our method of Expt. 2 are shown in Fig. 2 with red color, and the averages of 4 metrics of our method
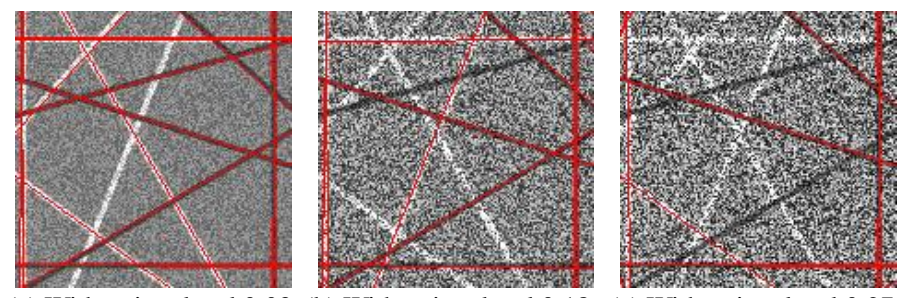

(a) With noises level 0.03 (b) With noises level 0.18 (c) With noises level 0.27

Fig. 1. Line detection results with different speckle noise levels using our method.

TABLE I. METRICS COMPARISON OF THE 3 METHODS IN EXPT. 1

\begin{tabular}{cccc}
\hline & RT-based Method & NHT-based Method & Our Method \\
\hline FA & 2.67 & 3.00 & $\mathbf{1 . 6 7}$ \\
MD & 4.67 & 5.67 & $\mathbf{3 . 6 7}$ \\
ND & 0.36 & 0.47 & $\mathbf{0 . 3 1}$ \\
RE & $\mathbf{2 3 . 1 0}$ & 89.73 & 29.98 \\
\hline
\end{tabular}
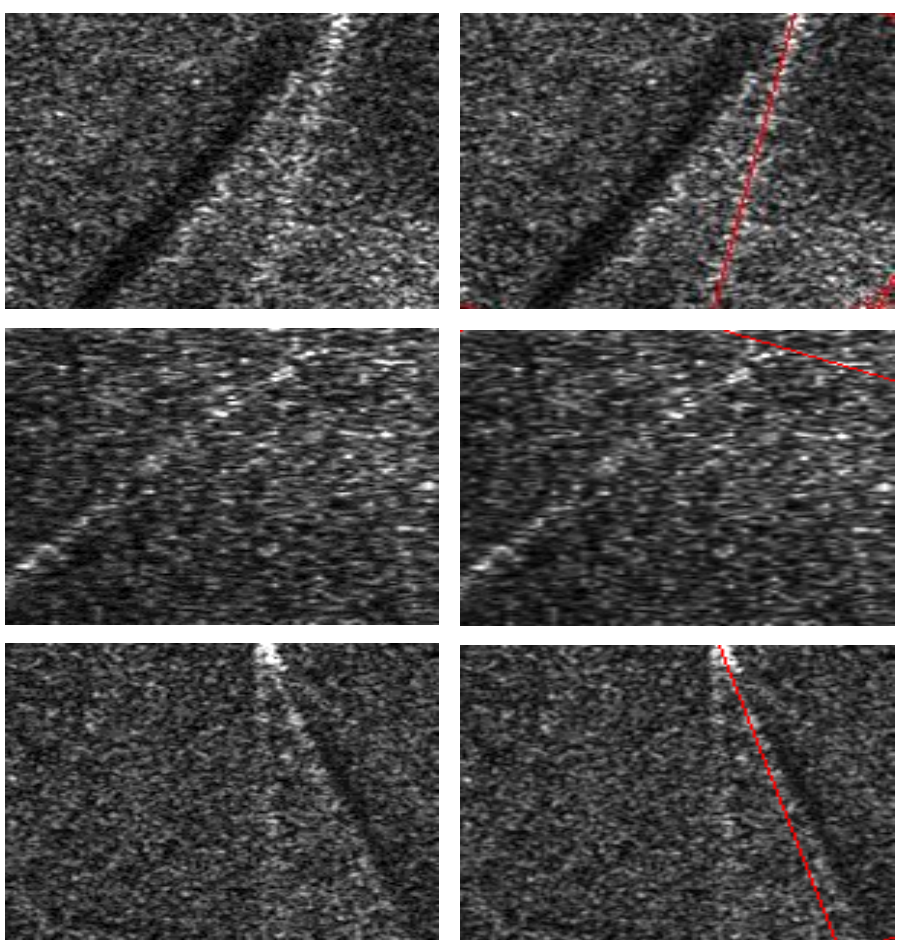

(a) Original images

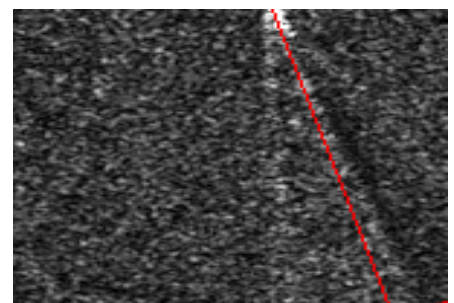

(b) RT based Method

and other 2 methods are listed in Table II. For Expt. 3, the radius of clustering is still set to 20 pixels, but the averages of all 58 FTs are different with each other. For all of the 58 real SAR images in this experiment, the averages of 4 indices of our method and other 2 methods are listed in Table III, and some representative results of all the 3 methods are shown in Fig. 3 with red color.

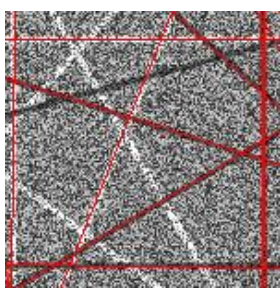

(a) With line width 3

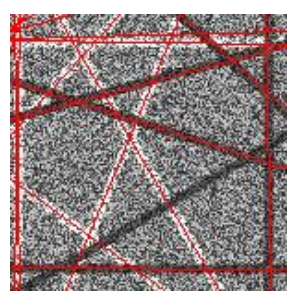

(b) With line width 5

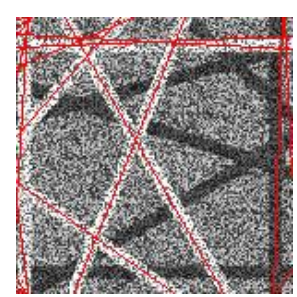

(c) With line width 7

Fig. 2. Line detection results with different line widths using our method.

TABLE II. METRICS COMPARISON OF THE 3 METHODS IN EXPT. 2

\begin{tabular}{cccc}
\hline & RT-based Method & NHT-based Method & Our Method \\
\hline FA & 6.50 & 8.00 & $\mathbf{5 . 6 7}$ \\
MD & 6.50 & 6.67 & $\mathbf{4 . 0 0}$ \\
ND & 0.70 & 0.56 & $\mathbf{0 . 3 4}$ \\
RE & $\mathbf{2 3 . 5 6}$ & 90.79 & 31.48 \\
\hline
\end{tabular}
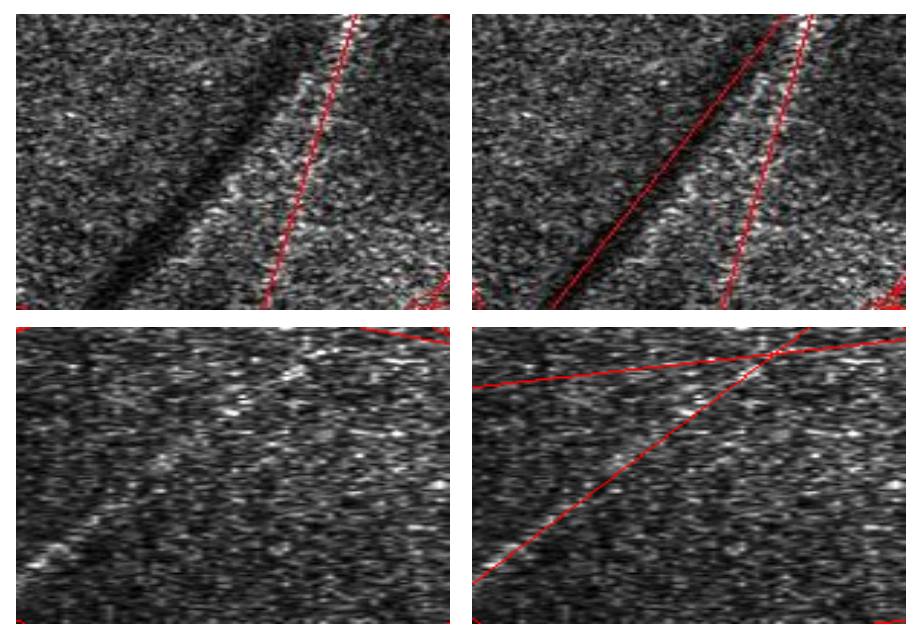

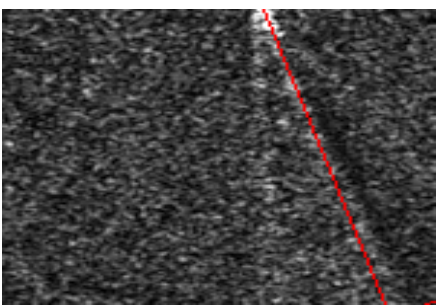

(c) NHT based Method

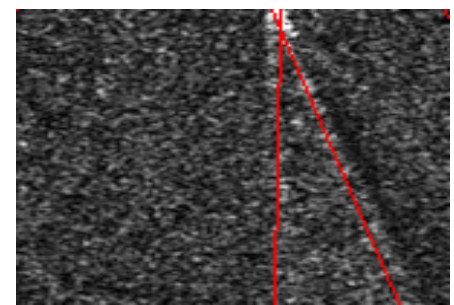

(d) Our Method

Fig. 3.Some ship wakes detection results of the 58 real SAR images using different methods. 
TABLE III. METRICS COMPARISON OF THE 3 METHODS IN EXPT. 3

\begin{tabular}{cccc}
\hline & RT-based Method & NHT-based Method & Our Method \\
\hline FA & 1.93 & 2.35 & $\mathbf{1 . 9 0}$ \\
MD & 0.26 & 0.38 & $\mathbf{0 . 0 0}$ \\
ND & 0.25 & 0.37 & $\mathbf{0 . 0 1}$ \\
RE & $\mathbf{2 3 . 9 2}$ & 94.31 & 28.77 \\
\hline
\end{tabular}

In the above tables, the best index among the 3 comparison methods is shown in bold. From Table I, we see that FA, MD, and ND indices of our method are the smallest than those of other 2 methods, which means that our method is more antinoise than other methods. In Table II, FA, MD and ND indices of our method are still the smallest than those of other 2 methods, which represents that our method is more insensitive to the line width than other methods. In Table III, FA, MD, and ND indices of our method are also the smallest than those of other 2 methods, which implies that our method is better than other methods under the real conditions. In addition to these, seen from all the 3 tables, the runtime efficiency of our method is slightly slower than that of the RT based method, but markedly faster than that of the NHT based method.

\section{CONCLUSIONS}

In this paper, a new GPCT based ship wakes detection method for SAR images is proposed. Here, GPCT is an improvement to the traditional PCT, which extends the binary transformation into grayscale transformation, and can deal with grayscale SAR images directly. Experimental results both using synthetic and real SAR images show that, the anti-noise performance and detective accuracy of our new method are excellent, and at the same time, our method is more insensitive to the width of ship wakes. The runtime efficiency of our method is slightly slower than the RT based method, and markedly faster than the NHT based method.

\section{REFERENCES}

[1] M. T. Rey, J. K. Tunaley, J. T. Folinsbee, P. A. Jahans, J. A. Dixon, and M. R. Vant, "Application Of Radon Transform Techniques To Wake Detection In Seasat-A SAR Images," IEEE Trans. Geosci. Remote Sens., vol. 28, no. 4, pp. 553-560, 1990.

[2] J.-S. Chong and M.-H. Zhu, "Ship Wake Detection Algorithm in SAR Image Based on Normalized Grey Level Hough
Transform," Journal of Image and Graphics, vol. 9, no. 2, pp. 146-150, Feb. 2004.

[3] N.-N. Liu, J.-W. Liu, and Y.-F. Cui, "Cluster-Based Split-Window Radon Transform Algorithm for Ship Wake Detection," in 2009 WRI World Congress on Computer Science and Information Engineering, vol. 5, pp. 773-777.

[4] J.-Q. Ai, X.-Y. Qi, W.-D. Yu, Y.-K. Deng, F. Liu, L. Shi, and Y.-F. Jia, “A Novel Ship Wake CFAR Detection Algorithm Based on SCR Enhancement and Normalized Hough Transform," IEEE Geosci. Remote Sens. Lett., vol. 8, no. 4, pp. 681-685, 2011.

[5] A. Inselberg, A. Chatterjee, and B. Dimsdale, "System using parallel coordinates for automated line detection in noisy images,” U.S. Patent 563198220, May 1997.

[6] S. El Mejdani, R. Egli, and F. Dubeau, "Old and new straight-line detectors: Description and comparison," Patt. Recognit., vol. 41, no. 6, pp. 1845-1866, Jun. 2008.

[7] R. Lo and W.-H. Tsai, "Gray-scale hough transform for thick line detection in gray-scale images," Patt. Recognit., vol. 28, no. 5, pp. 647-661, May 1995.

[8] T. Kurita, "An efficient agglomerative clustering algorithm using a heap," Patt. Recognit., vol. 24, no. 3, pp. 205-209, 1991.

[9] J.-L. Fan and B. Lei, "A modified valley-emphasis method for automatic thresholding," Patt. Recognit. Lett., vol. 33, no. 6, pp. 703-708, Apr. 2012. 